\title{
Gravimetrische Bestimmung des Salpeters in Fleisch.
}

\author{
Von \\ C. Paal und Gustav Mehrtens.
}

\section{Mitteilung ans dem Laboratorium für angewandte Chemie an der Universität Erlangen.}

Die Untersuchungen von K. Farnsteiner ${ }^{1}$ ) und W. Stüber ${ }^{2}$ ) ergaben, daß von den bisher vorgeschlagenen und angewandten Methoden zur Bestimmung von Salpeter in Fleisch und Fleischwaren nur das gasvolumetrische Verfahren der Überführung des Nitrats in Stickoxyd zuverlässige Ergebnisse liefert, während die beiden anderen, ebenfalls in den "Vereinbarungen" angeführten, auf der Reduktion des Nitrats in saurer oder alkaliseher Lösung zu Ammoniak beruhenden Verfahren mit beträchtlichen Fehlern verbunden sind. Nachdem vor etwas mekr als 2 Jahren von M. $\mathrm{Busch}^{8}$ ) ein ebenso bequemes wie genaues Verfahren zur gewichtsanalytischen Bestimmung von Salpetersäure und Nitraten aufgefunden war, lag es nahe, dieses Verfahren auch auf seine Brauchbarkeit zum qualitativen und quantitativen Nachweis von Salpeter in Fleisch und Fleischwaren zu prüfen. Bekanntlich beruht diese Methode auf der Fällbarkeit Salpetersäure oder Nitrate enthaltender Flüssigkeiten mittels einer essigsauren Lösung von „Nitron", der synthetischen Base Diphen ylendanilodihydrotriazol, deren salpetersaures Salz so schwerlöslich ist, dafo es bei $0^{0}$ noch in einer Verdünnung von 1:133000 auskrystallisiert. ${ }^{4}$ )

Das Verfahren hat sich bisher in allen Fällen gut bewährt ${ }^{5}$ ), so zur Bestimmung unorganischer und organischer Nitrate, zum Nachweis kleiner Mengen von Nitraten in Wasser, zur Trennung von Nitraten und Nitriten und zur Analyse von organischen Salpetersäureestern.

Wie schon von $\mathrm{M}$. $\mathrm{Bu} s \mathrm{ch}$ nachgewiesen wurde, gibt das Nitron nicht nur mit Salpetersäure, sondern auch mit einigen anderen Säuren mehr oder minder schwerlösliche Salze. So ist z. B. das salzsaure Nitron in Wasser nicht gerade leicht löslich. Es war daher zu prüfen, ob das in Fleisch und Fleischwaren stets in mehr oder minder beträchtlicher Menge vorhandene Kochsalz die Anwendung der Nitron-Methode zur Bestimmung von Salpeter in diesen Nahrungsmitteln gestatten würde. Auch die Extraktivstoffe des Fleisches konnten möglicherweise das Verfahren in ungünstigem Sinne beeinflussen.

Für unsere Versuche verwendeten wir von der Firma E. Merck-Darmstadt bezogenes Nitron und zwar wurde nach der Vorschrift von M. Busch eine $10^{\circ} \%$-ige

1) Diese Zeitschrift 1905, 10, 329.

2) Diese Zeitschrift 1905, 10, 330.

3) Ber. Deutsch. Chem. Gesellsch. 1905, 38, 861.

$\left.{ }^{4}\right)$ Ber. Deutsch. Chem. Gesellsch. 1905, 38, 4055 .

8) Vergl. A. Gutbier: Zeitschr. angew. Chem. 1905, 18, 1681.

II. B usch: Diese Zeitschr. 1905, 9, 464.

Paal und Weidenkaff: Ber. Deutsch. Chem. Gesellsch. 1905, 38, 1688.

E. Fischer: Ber. Deutsch. Chem. Gesellsch. 1905, 38, 4190.

W. Traube und A. Biltz: Ber. Deutsch. Chem. Gesellsch. 1906, 39, 168.

Lunge und Berl: Zeitschr. angew. Chem. 1905, 18, 1681.

MI. Busch: Ber. Deutseh. Chem. Gesellsch. 1906, 39, 1401.

M. Busch: Zeitschr. für das gesamte Schiek- und Sprengstoffwesen 1906, 232. 
Lösung von Nitron in 5\%-iger Essigsäure dargestellt. Zur Prüfung auf die Reinheit des Präparates wurde eine Nitratbestimmung von chemisch reinem Kalisalpeter mittels dieser Nitronlösung ausgeführt:

$10 \mathrm{ccm}$ der 1\%-igen Kaliumnitrat-Lösung lieferten 0,3718 $\mathrm{g}$ Nitronnitrat.

$$
\text { Berechnet: Gefunden: }
$$

Kaliumnitrat $0,1 \mathrm{~g} \quad 0,1 \mathrm{~g}$

Um einen etwaigen schädlichen Einfluß der Extraktivstoffe des Fleisches festzustellen, wurde eine kleine Menge einer nitratfreien Fleischabkochung mit dem gleichen Volumen der essigsauren Nitronlösung versetzt. Es entstand nach längerem Stehen der Mischung nur eine gexinge Trübung.

Ein quantitativer Versuch, bei welchem 100 cem obiger Fleischabkochung mit $25 \mathrm{ccm}$ der 1\%-igen Kaliumnitrat-Lösung und dann mit der Nitronlösung versetzt wurden, lieferte kein befriedigendes Ergebnis. Aus der heißen Mischung kristallisierte das Nitronnitrat nicht in den charakteristischen, schönen, weißen Nadeln, sondern in klumpig zusammenhängenden Blättchen aus, die sich nur schwer im Neubauer-Tiegel absaugen lieben und ihre braune Farbe auch nach dem Auswaschen mit $10 \mathrm{ccm}$ Eiswasser nicht verloren. Der Niederschlag wog $0,9545 \mathrm{~g}$, woraus sich $0,257 \mathrm{~g}$ Kaliumnitrat berechnen, während nur $0,25 \mathrm{~g}$ in der Lösung vorhanden waren. Die Nitronfällung war somit nicht rein, sondern hatte, wie schon aus der Farbe ersichtlich war, aus der Fleischabkochung irgendwelche gefärbte, amorphe Stoffe aufgenommen.

Es handelte sich also darum, diese das Nitronnitrat verunreinigenden Substanzen aus der Fleischabkochung vor dem Zusatz der Nitronlösung in geeigneter Weise zu entfernen. Wenn auch die eigentlichen Eiweißkörper beim Auskochen des Fleisches koagulieren, so gehen doch Leim, Albumosen und verschiedene andere lösliche Bestandteile des Fleisches in die Auskochung über, die von dem auskrystallisierenden Nitronnitrat aufgenommen werden konnten. Wir fanden im Bleiacetat, das in $10 \%$ iger Lösung angewendet wurde, das geeignete Mittel zur Entfernung der die Nitronfällung verunreinigenden Stoffe. Die Verwendung dieses Reagens bot noch den weiteren Vorteil, daß bei Anwesenheit größerer Mengen Chlornatrium in den zu untersuchenden Fleischproben auch das Chlor zum gröBten Teil ausgeschieden wurde. Wenn auch das Nitronchlorhydrat nicht eben schwerlöslich ist, so kann sich doch ein beträchtlicher Kochsalzgehalt, wenn es sich um die Bestimmung geringer Mengen Salpeter handelt, recht störend bemerkbar machen. Schon M. Busch hat in seiner Mitteilung über „Bestimmung der Salpetersäure in Wasser “1) darauf hingewiesen, daß ein das Verhältnis 1:250 überschreitender Gehalt von Chlornatrium das Analysenergebnis durch Ausscheidung von Nitronchlorhydrat ungünstig beeinflussen kann. Letzteres ist allerdings durch die gelatinöse Form, in der es sich ausscheidet, leicht zu erkennen.

Gerade bei Fleisch und Fleischwaren, die oft einen erheblichen Kochsalzzusatz erhalten, schien es daher geboten, vor der Nitratbestimmung das Chlornatrium, bezw, die Chlor-Ionen nach Möglichkeit zu entfernen. Aus diesem Grunde wandten wir bei den folgenden Versuchen basisches Bleiacetat in Form von Bleiessig an, da dieser die Chlor-Ionen als basisches Bleichlorid fast vollständig ausfällt. Nach der Fällung wurde das Filtrat mit Essigsäure angesäuert, weil auch das Nitron durch basisches 
Bleiacetat, nicht aber durch das neutrale Acetat gefällt wird. Die Ausführung der Analysen geschah in folgender Weise:

100 ccm Fleischabkochung, mit oder ohne Kochsalzzusatz, wurden nach Zugabe bestimmter Mengen reiner Kaliumnitrat-Lösung solange mit Bleiessig versetzt, als noch Fällung eintrat und dann kurze Zeit zum schwachen Sieden erhitzt, wobei sich der ziemlich voluminöse Niederschlag zusammenballt. Nach dem Erkalten wurde filtriert, der Niederschlag gut ausgewaschen, das Filtrat bis nahe zum Sieden erhitzt, mit Essigsäure angesäuert und $10 \mathrm{ccm}$ - bei größerem Nitratgehalt entsprechend mehr - der schon erwähnten Nitronlösung zugegeben. Nach 21/8-3-stiündigem Stehen der Flüssigkeit in Eis oder Eiswasser wurden die ausgeschiedenen Nadeln des Nitronnitrats in einem gewogenen Neubauer-oder Gooch-Tiegel - auch ein Asbestfilterrohr ist dazu geeignet - mit der Saugpumpe abfiltriert und dann in kleinen Anteilen mit im ganzen $10 \mathrm{ccm}$ Eiswasser ausgewaschen. Den Tiegel samt Niederschlag trocknet man hierauf bei $110^{\circ}$ bis zum konstanten Gewicht, was ungefähr 1 Stunde erfordert.

Die Berechnung des Kaliumnitrats aus dem Nitronnitrat geschieht nach folgendem Ansatz:

$$
x=\frac{a .101}{375}
$$

$\mathbf{a}=$ Gewicht des Nitronnitrats

$101=$ Molekulargewicht des Kaliumnitrats

$375=$ Molekulargewicht des Nitronnitrats.

Tabelle I.

\begin{tabular}{|c|c|c|c|c|c|c|}
\hline \multirow{2}{*}{$\begin{array}{l}\text { Versuch } \\
\text { No. }\end{array}$} & \multirow{2}{*}{$\begin{array}{l}\text { Fleisch- } \\
\text { Abkochung }\end{array}$} & \multirow{2}{*}{$\begin{array}{c}\text { 1\%/n-ige Kalium- } \\
\text { nitratiösung } \\
\text { zugesetzt }\end{array}$} & \multirow{2}{*}{$\begin{array}{l}\text { Chlornatrium- } \\
\text { Zusatz }\end{array}$} & \multirow{2}{*}{$\begin{array}{l}\text { Gewicht des } \\
\text { Nitronnitrats }\end{array}$} & \multicolumn{2}{|c|}{ Kaliumnitrat } \\
\hline & & & & & gefunden & $\begin{array}{l}\text { in der Lösung } \\
\text { vorhanden }\end{array}$ \\
\hline I & $100 \mathrm{ccm}$ & $5 \mathrm{ccm}$ & $1,0 \mathrm{~g}$ & $0,1625 \mathrm{~g}$ & $0,044 \mathrm{~g}$ & $0,05 \mathrm{~g}$ \\
\hline II & 100 & 6 & 1,0 & 0,2095 & 0,056 & 0,06 \\
\hline III & 100 & 5 & 1,0 & 0,1686 & 0,045 & 0,05 \\
\hline IV & 100 & 5 & 1,0 n & 0,1575 & 0,042 & 0,05 \\
\hline $\mathrm{V}$ & 100 & 5 & 1,0 & 0,1463 & 0,040 & 0,05 , \\
\hline
\end{tabular}

Die Versuche wurden insofern etwas variiert, als bei den Versuchen No. I, II und III die Bleiessigfällungen mit kaltem, bei No. IV und $V$ mit heißem Wasser ausgewaschen wurden. Bei Versuch No. V hatte man auferdem zur Vermeidung eines Überschusses die Bleilösung aus einer Bürette zufließen lassen, um zu ermitteln, ob etwa ein Überschuß des Fällungsmittels ungünstig wirke. Alle Bleifällungen gaben trotz sorgfältigen Auswaschens deutliche Nitratreaktion mit Diphenylamin, woraus sich die durchgehends zu niedrigen Analysenergebnisse erklären. $\mathrm{Ob}$ das Zurückhalten von Nitrat durch die Bleiessigfällung auf der Bildung eines schwerlöslichen, basischen Bleinitrates, oder, was wir für wahrscheinlicher halten, auf Adsorption beruht, muß dahingestellt bleiben.

Wir wandten daher in der Folge statt des basischen neutrales Bleiacetat (Bleizucker) an und erhielten so durchwegs sehr zufriedenstellende Analysenergebnisse. Hat eine in der Fleischabkochung mit Silbernitrat und Salpetersäure angestellte, qualitative Vorprobe die Anwesenheit größerer Mengen Kochsalz ergeben, so engt man 
den für die Nitratbestimmung verwendeten Teil des Extraktes - gewöhnlich $100 \mathrm{ccm}$, bei sehr geringen Nitratmengen mehr - auf dem Wasserbade auf etwa $20 \mathrm{ccm}$ ein und versetzt nach dem Erkalten mit neutraler Bleiacetatlösung, $\left[\mathrm{Pb}_{2}\left(\mathrm{C}_{2} \mathrm{H}_{3} \mathrm{O}_{2}\right)_{2}\right]$, so lange noch Fällung entsteht. Nach kurzem Erhitzen und Absitzenlassen des Niederschlages bis zum völligen Erkalten wurde filtriert, ausgewaschen und das Filtrat auf 150 bis $180 \mathrm{ccm}$, bei sehr wenig Nitrat nur auf $100 \mathrm{ccm}$, mit Wasser verdünnt. Die Fällung des Nitrates als Nitronnitrat geschieht dann in der schon angegebenen Weise.

Bei dem manchmal erforderlichen, mehrnaligen starken Auskochen der Fleischproben beobachtete man öfters, daß die Flüssigkeit nach der Bleiacetatfüllung sehr langsam filtriert. Diesem Übelstande kann leicht dadurch abgeholfen werden, daß man der eingeengten Fleischabkochung vor der Fällung mit Bleiacetat 2-3 Tropfen Ammoniak zusetzt. Ein größerer Ammoniakzusatz jedoch muß vermieden werden, weil dadurch sonst Verbältnisse geschaffen werden, wie sie bei Anwendung basischen Bleiacetats bestehen; nämlich, daß Nitrat im Bleiniederschlage zurückgehalten wird.

Bei den folgenden Versuchen ohne und mit Kochsalzzusatz wurde letzterer mit Absicht groß gewählt, um die Brauchbarkeit der Methode auch bei hohem ChlornatriumGehalt der Fleischproben, z. B. bei Pöckelfleisch, zu erproben, $1 \mathrm{~g}$ Chlornatrium in $100 \mathrm{ccm}$ Fleischabkochung entsprieht ungefähr 10\% Kochsalz in der betreffenden Fleischprobe, da zum vollständigen Extrahieren des Salpeters auf $50 \mathrm{~g}$ Fleisch kaum mehr als $500 \mathrm{ccm}$ Wasser erforderlich sind.

Tabelle II.

A. Ohne Kochsalzzusatz.

\begin{tabular}{|c|c|c|c|c|c|}
\hline \multirow{2}{*}{$\begin{array}{l}\text { Versuch } \\
\text { No. }\end{array}$} & \multirow{2}{*}{$\begin{array}{l}\text { Fleiseh- } \\
\text { abkochung }\end{array}$} & \multirow{2}{*}{$\begin{array}{l}1 \% \text {-ige Kalium- } \\
\text { nitratiosung } \\
\text { zugesetzt }\end{array}$} & \multirow{2}{*}{$\begin{array}{l}\text { Gewieht des } \\
\text { Nitronnitrats }\end{array}$} & \multicolumn{2}{|c|}{ Kaliumnitrat } \\
\hline & & & & gefunden & $\begin{array}{l}\text { in der luösung } \\
\text { rorhanden }\end{array}$ \\
\hline I & $100 \mathrm{ccm}$ & $10 \mathrm{ccm}$ & $0,3707 \mathrm{~g}$ & $0,0988 \mathrm{~g}$ & $0,10 \mathrm{~g}$ \\
\hline II & $\pi$ & 8, & 0,2970 n & 0,0799 & 0,08 , \\
\hline III & , & 5 & 0,1858 \# & 0,0500 & 0,05, \\
\hline IV & & $1 *$ & 0,0352 * & 0,0095 & 0,01 \\
\hline
\end{tabular}

B. Mit Kochsalzzusatz.

\begin{tabular}{|c|c|c|c|c|c|c|}
\hline \multirow{2}{*}{$\begin{array}{l}\text { Versueh } \\
\text { No. }\end{array}$} & \multirow{2}{*}{$\begin{array}{l}\text { Fleisch- } \\
\text { abkochung }\end{array}$} & \multirow{2}{*}{$\begin{array}{l}\text { Chlornatrium- } \\
\text { Znsatz }\end{array}$} & \multirow{2}{*}{$\begin{array}{c}1 \% \text {-ige Kalium- } \\
\text { nitratlösung } \\
\text { zugesetzt }\end{array}$} & \multirow{2}{*}{$\begin{array}{l}\text { Gewieht des } \\
\text { Nitronnitrats }\end{array}$} & \multicolumn{2}{|c|}{ Kaliumnitrat } \\
\hline & & & & & gefunden & $\begin{array}{l}\text { in der Lösung } \\
\text { vorhanden }\end{array}$ \\
\hline I & $100 \mathrm{ccm}$ & $1,0 \mathrm{~g}$ & $20 \mathrm{~cm}$ & $0,7467 \mathrm{~g}$ & $0,200 \mathrm{~g}$ & $0,20 \mathrm{~g}$ \\
\hline II & y & 0,6 , & 15, & 0,5560 & 0,149 " & 0,15 \\
\hline III & 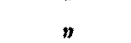 & 1,0 & 10 & 0,3800 & 0,102 , & 0,10, \\
\hline 17 & , & 1,0 & 5 & 0,1890 & 0,051 , & 0,05 n \\
\hline$\nabla$ & , & 1,0 \# & 3 & 0,1078 , & 0,029 n & 0,03 \\
\hline VI & $n$ & 1,0 , & 1 & $0,0345 n$ & 0,009 , & 0,01 n \\
\hline
\end{tabular}

Wie aus den vorstehenden Tabellen ersichtlich ist, liefert die Methode auch bei großen Kochsalzmengen recht gute Ergebnisse; so beträgt bei der Analyse B, No. VI, der Kochsalzgehalt das Hundertfache vọn dem des Salpeters. 
Bei den Analysen A, No. V und B, No VI wurde das Filtrat vom Bleiacetatniederschlag mit Wasser nur auf $100 \mathrm{ccm}$ gebracht. Bei den Bestimmungen B, No. IV und B, No. VI schieden sich nach der Fällung mit Nitron beim Erkalten neben den Nadeln des Nitronnitrats auch gallertige Flöckchen des Nitronchlorhydrates aus. Die Flüssigkeit wurde daher nochmals erwärmt, bis sich das Chlorhydrat wieder gelöst hatte und noch 20-30 ccm Wasser zugegeben. Dann ließ man wieder in Eiswasser erkalten, worauf sich reines Nitronnitrat abschied. Beide Analysen lieferten genaue Ergebnisse. Eine etwaige Abscheidung von Nitronchlorhydrat ist daher auf diese Weise leicht za beseitigen.

Für die folgenden Versuche wurde fein gehacktes Rindfleisch mit bestimmten Mengen reinen Kalisalpeters oder 1\%-iger Kaliumnitrat-Lösung vermischt und dann wurden die Fleischproben bis zum Verschwinden der Diphenylaminreaktion mit destilliertem Wasser ausgekocht. Das Extrahieren geschieht zweckmäfig in der Weise, daß man zuerst 1-2 Stunden unter häufigem Umschütteln mit lauwarmem Wasser die Fleischmasse digeriert, dann erst aufkocht und nun mit kleinen Mengen Wasser unter stetigem Erhitzen bis zum Verschwinden der Diphenylaminreaktion auslaugt. $50 \mathrm{~g}$ Fleisch kann auf diese Weise mit $500 \mathrm{ccm}$ Wasser der Salpetergehalt vollständig entzogen werden.

Tabelle III.

\begin{tabular}{|c|c|c|c|c|c|c|c|c|c|}
\hline \multirow{2}{*}{$\begin{array}{l}\text { Versueh } \\
\text { No. }\end{array}$} & \multirow{2}{*}{$\begin{array}{l}\text { Fleiseh- } \\
\text { menge }\end{array}$} & \multirow{2}{*}{$\begin{array}{l}\text { Kalium- } \\
\text { nitrat- } \\
\text { Zusatz }\end{array}$} & \multirow{2}{*}{$\begin{array}{l}\text { Volumen } \\
\text { der Aus- } \\
\text { koehung }\end{array}$} & \multirow{2}{*}{$\begin{array}{c}\text { Zur Nitrat- } \\
\text { bestimmung } \\
\text { verwendet }\end{array}$} & \multirow{2}{*}{$\begin{array}{c}\text { Nitron- } \\
\text { nitrat }\end{array}$} & \multicolumn{2}{|c|}{ Kaliumnitrat } & \multicolumn{2}{|c|}{$\begin{array}{l}\text { Gesamtmenge des } \\
\text { Kaliumnitrats im Fleiseh }\end{array}$} \\
\hline & & & & & & gefunden & bereehnet & gefunden & berechnet \\
\hline$I$ & $50 \mathrm{~g}$ & $\begin{array}{l}20 \mathrm{ccm} \\
1 \% \text {-ige } \\
\text { Lösung }\end{array}$ & $500 \mathrm{cem}$ & $250 \mathrm{ccm}$ & $0,3401 \mathrm{~g}$ & $0,092 \mathrm{~g}$ & $0,100 \mathrm{~g}$ & $0,184 \mathrm{~g}$ & $0,2000 \mathrm{~g}$ \\
\hline $\begin{array}{l}\text { II a } \\
\text { II b }\end{array}$ & \} $50 \mathrm{~g}$ & $\begin{array}{l}30 \text { ccm } \\
1^{0} / 0 \text {-ige } \\
\text { Losung }\end{array}$ & $500 \mathrm{ccm}$ & $\begin{array}{l}75 \mathrm{ccm} \\
50\end{array}$ & $\begin{array}{l}0,1617 \mathrm{~g} \\
0,1087\end{array}$ & $\begin{array}{l}0,044 \mathrm{~g} \\
0,029\end{array}$ & $\begin{array}{l}0,045 \mathrm{~g} \\
0,080\end{array}$ & $\begin{array}{l}0,2938 \mathrm{~g} \\
0,2900^{n}\end{array}$ & $0,3000 \mathrm{~g}$ \\
\hline III & $50 \mathrm{~g}$ & $0,3132 \mathrm{~g}$ & $500 \mathrm{ccm}$ & $100 \mathrm{ccm}$ & $0,2288 \mathrm{~g}$ & $0,0616 \mathrm{~g}$ & $0,0626 \mathrm{~g}$ & $0,308 \mathrm{~g}$ & $0,3132 \mathrm{~g}$ \\
\hline
\end{tabular}

Um unser gewichtsanalytisches Verfahren mit der von Stüber benutzten Schlösing-Wagner'schen volumetrischen Bestimmung des Nitrates als Stickoxyd zu vergleichen, haben wir die folgenden Analysen nach letzterem Verfahren ausgeführt.

Bei versuchsweise ausgeführten Bestimmungen des Kaliumnitrat-Gehaltes reiner Salpeterlösungen unter genauer Einhaltung der von Stüber gegebenen Vorschrift ${ }^{1}$ ) wurde der Nitrongehalt stets etwas zu hoch gefunden, eine Beobachtung, welche im hiesigen Institut auch häufig bei Benutzung des Schulze-Tiemann'schen Verfahrens gemacht wurde. Weiter ergab sich, daß es nicht von großem Belang ist, ob die Ablesung des Gasvolumens im Azotometer nach 11/2, 2 oder 3 Stunden erfolgt,

1) An Stelle des von Stäber aus einem Allihn'sehen Röhrehen mit Quetsehbahn hergestellten Tropftrichters wurde ein solcher mit eingeschliffenem Glashahn verwendet, was den Vorteil bietet, daß3 man genau beobachten kann, ob bei stärkerem Druck im Kolben StickoxydBlässchen in das 'I'richterrohr eingetreten sind. 
denn die Volumenänderungen waren zu gering, um für die Praxis in Betracht zu kommen. Selbst nach 4-stündigem Ablesen konnte in keinem Falle eine nennenswerthe Abnahme des Gasvolumens festgestellt werden.

Tabelle IV.

Nitrat-Bestimmungen nach Schlösing-Wagner:

\begin{tabular}{|c|c|c|c|c|c|c|}
\hline \multirow{2}{*}{ No. } & \multirow{2}{*}{$\begin{array}{l}\text { Angewendete } \\
1 \% \text {-ige } \\
\text { Kallumnitrat- } \\
\text { Lösung }\end{array}$} & \multirow{2}{*}{$\begin{array}{c}\text { Stickoxydgas - } \\
\text { Volumen }{ }^{1)}\end{array}$} & \multirow{2}{*}{$\begin{array}{l}\text { Barometer- } \\
\text { stand }\end{array}$} & \multirow{2}{*}{ Temperatur } & \multicolumn{2}{|c|}{ Kaliumnitrat } \\
\hline & & & & & gefunden & berechnet \\
\hline 1 & $10 \mathrm{ccm}$ & $26,1 \mathrm{ccm}$ & $733 \mathrm{~mm}$ & 170 & $0,104 \mathrm{~g}$ & $0,10 \mathrm{~g}$ \\
\hline II & 5 & 12,8 \# & 733 & $15^{0}$ & 0,052 & 0,05 \\
\hline
\end{tabular}

1) Das Gasvolumen wurde nach $1^{1 / 2}$ Stunden abgelesen.

$50 \mathrm{~g}$ Hackfleisch wurden mit 0,5 Kaliumnitrat verrieben, bis zum Verschwinden der Diphenylaminreaktion mit Wasser in der oben beschriebenen Weise ausgelaugt und die wässerige Lösung auf 1 Liter verdünnt. In abgemessenen Mengen dieser Lösung wurde der Nitratgehalt nach Sehlösing-Wagner und nach der Nitronmethode bestimmt:

Tabelle V.

A. Schlösing-Wagner'sche Methode.

\begin{tabular}{|c|c|c|c|c|c|c|c|c|c|}
\hline \multirow{2}{*}{ No. } & \multirow{2}{*}{$\begin{array}{l}\text { Yolumen } \\
\text { der Fleiseh- } \\
\text { ablocolung }\end{array}$} & \multirow{2}{*}{$\begin{array}{c}\text { Ablosung } \\
\text { des Gas- } \\
\text { rolumens } \\
\text { naeh }\end{array}$} & \multirow{2}{*}{$\begin{array}{l}\text { Stickoxyd- } \\
\text { Volumen }\end{array}$} & \multirow{2}{*}{$\begin{array}{l}\text { Barometer- } \\
\text { stand }\end{array}$} & \multirow{2}{*}{$\begin{array}{l}\text { Tempe- } \\
\text { ratur }\end{array}$} & \multicolumn{2}{|c|}{ Kalnmnituat } & \multicolumn{2}{|c|}{$\begin{array}{c}\text { Gesamtmenge des } \\
\text { Kaliumnitrats im Fleisch }\end{array}$} \\
\hline & & & & & & gefunden & berechnet & gefunden & berechnet \\
\hline I & $150 \mathrm{ccm}$ & $\begin{array}{l}1 / 2 \text { Stdn. } \\
3\end{array}$ & $\begin{array}{l}19,0 \mathrm{cem} \\
18,8\end{array}$ & $\begin{array}{c}738 \mathrm{~mm} \\
n\end{array}$ & $\begin{array}{c}16,5^{0} \\
16^{\circ}\end{array}$ & $\begin{array}{l}0,0758 \mathrm{~g} \\
0,0757\end{array}$ & $\begin{array}{c}0,075 \mathrm{~g} \\
\end{array}$ & $\begin{array}{l}0,5053 \mathrm{~g} \\
0,5047\end{array}$ & $\begin{array}{c}0,5 \mathrm{~g} \\
\Rightarrow\end{array}$ \\
\hline II & $100 \mathrm{ccm}$ & $\begin{array}{l}\mathbf{1}^{1 / 2} \text { Stdn. } \\
3\end{array}$ & $\begin{array}{l}12,2 \mathrm{~cm} \\
12,0 \text { n }\end{array}$ & $\begin{array}{c}730 \mathrm{~mm} \\
\text {, }\end{array}$ & $\begin{array}{l}16^{\circ} \\
16^{\circ}\end{array}$ & $\begin{array}{l}0,049 \mathrm{~g} \\
0,048\end{array}$ & $\begin{array}{c}0,05 \mathrm{~g} \\
\end{array}$ & $\begin{array}{l}0,49 \mathrm{~g} \\
0,48\end{array}$ & $\begin{array}{c}0,5 \mathrm{~g} \\
\end{array}$ \\
\hline
\end{tabular}

B. Nitron-Methode.

\begin{tabular}{|c|c|c|c|c|c|c|}
\hline \multirow{2}{*}{ No. } & \multirow{2}{*}{$\begin{array}{c}\text { Volumen } \\
\text { der Fleiseh } \\
\text { abkochung }\end{array}$} & \multirow{2}{*}{$\begin{array}{l}\text { Gewicht des } \\
\text { Nitronnitrats }\end{array}$} & \multicolumn{2}{|c|}{ Kaliumnitrat } & \multicolumn{2}{|c|}{$\begin{array}{c}\text { Gesamtmenge des } \\
\text { Kaliumnitrats im Fleisch }\end{array}$} \\
\hline & & & gefunden & berechnet & gefinden & beroehnet \\
\hline I & $150 \mathrm{ccm}$ & $0,2745 \mathrm{~g}$ & $0,0739 \mathrm{~g}$ & $0,075 \mathrm{~g}$ & $0,493 \mathrm{~g}$ & $0,5 \mathrm{~g}$ \\
\hline 11 & 100 & 0,1810 & 0,0487 & 0,050 & 0,487 & $n$ \\
\hline
\end{tabular}

Um auch noch ein Beispiel aus der Praxis zu geben, führen wir nachstehend Salpeterbestimmungen in rohem Schinken an. Der Nitratgehalt wurde nach SchlösingWagner und nach der Nitron-Methode ermittelt.

$75 \mathrm{~g}$ gut zerkleinerter, roher Schinken wurde in der schon angegebenen Weise mit Wasser ausgelaugt und die Flüssigkeit auf ein Volumen von $500 \mathrm{cem}$ gebracht. 
a) $200 \mathrm{ccm}$ des Extrakts wurden anf etwa $15 \mathrm{ccm}$ eingeengt und die Menge des Kaliumnitrats als Stickoxyd bestimmt. Es wurden $11,9 \mathrm{ccm}$ Stickoxyd bei $\mathrm{T}=18^{\circ}$ und $\mathrm{B}=735 \mathrm{~mm}$ gefunden, die $0,049 \mathrm{~g}$ Kaliumnitrat entsprechen.

b) Weitere $200 \mathrm{ccm}$ der Abkochung wurden auf dem Wasserbade auf nngefaht $50 \mathrm{ccm}$ eingedampft. (Bei sehr hohem Chlornatrium-Gehalt ist, wie schon erwähnt, auf etwa 20-30 ccm einzuengen.) Nach dem Erkalten wurden 3 Tropfen Ammoniak und neutrale Bleiacetatlösung zugefügt, bis keine Fällung mehr erfolgte. Nach kurzem Erhitzen zum schwachen Sieden und Absitzenlassen der Fällung wurde die erkaltete Flussigkeit filtriert, der Niederschlag ausgewaschen und das Filtrat nach dem Ansäuern mit Essigsảure heif mit $10 \mathrm{ccm}$ der schon erwähnten essigsauren NitronLösung gefällt. Nach 3-stündigem Stehenlassen in Eiswasser wurden die Krystalle des Nitronnitrats im Neubauer-Tiegel abgesangt, mit $10 \mathrm{ccm}$ Eiswasser gewaschen und nach dem Trocknen bei $110^{\circ}$ zur Wägung gebracht. $200 \mathrm{ccm}$ des Extrakts lieferten $0,1684 \mathrm{~g}$ Nitronnitrat $=0,045 \mathrm{~g}$ Kaliumnitrat.

Daraus ergibt sich in $75 \mathrm{~g}$ der Probe an Kaliumnitrat:

nach der Schlösing-Wagner'schen Methode 0,1225 $\mathrm{g}=0,163 \%$

$$
\text { " Nitron-Methode } \quad 0,1125 \mathrm{~g}=0,150 \% \text {. }
$$

Die gravimetrische Nitron-Methode zur Bestimmung von Salpeter in Fleisch und Fleischwaren liefert somit für die Zwecke der Praxis hinreichend genaue Ergebnisse, die mit den durch die gas. volumetrische Analyse ermittelten Werten befriedigend übereinstimmen. Da ferner die Nitratbestimmungen mittels Nitrons keinerlei experimentelle Schwierigkeiten bieten und mit einfachen Laboratoriumshilfsmitteln ausführbar sind, so können wir dieses Verfahren, das sich, wie zahlreiche Versuche dargetan haben, zur Bestimmung des Nitrat-Ions im allgemeinen vorzüglich eignet, auch zur quantitativen Bestimmung des Salpeters in Fleisch und Fleischwaren empfehlen.

\title{
Luxemburger Naturweine des Jahrganges $1904^{1}$ ).
}

\author{
Von \\ Doktor-Ingenieur J. Weiwers.
}

Mitteilung aus dem Bakteriologischen Staatslaboratorium in Luxemburg.

Die in der nachfolgenden Tabelle aufgeführten Weine aus dem Jahrgange 1904 sind im Monat Januar 1906 als garantiert naturrein von der Großherzoglichen Wein. baukommission zur Untersuchung eingesandt worden. Letztere erfolgte in der Zeit vom Januar bis A pril d. Js. nach den in der Bekanntmachung des deutschen Reichskanzlers vom 25. Juni 1896 festgelegten Methoden.

Wie vorauszusehen war, sind die Analysenergebnisse dieses Jahrganges recht günstig ausgefallen. Infolge der heißen, trockenen Witterung, die im Sommer 1904 vorherrschte, kamen die Trauben meistens vollreif und an manchen Orten sogar edelfaul zur Lese. Die Mostgewichte waren demgemäß hohe; sie schwankten zwischen $70^{\circ}-80^{\circ}$ Oechsle, während der Säuregehalt für unsere Verhältnisse niedrig war; er

1) Von dem Jahrgange 1903 sind keine Untersuchungen ausgeführt worden, da dieser in Luxemburg eine völlige Mißernte brachte. 